\title{
Inflammatory Alterations of the Extracellular Matrix in the Tumor Microenvironment
}

\author{
Junko Iijima ${ }^{1}$, Kenjiro Konno ${ }^{2}$ and Naoki Itano ${ }^{1, *}$
}

1 Department of Molecular Biosciences, Faculty of Life Sciences, Kyoto Sangyo University, Motoyama, Kamigamo, Kita-Ku, Kyoto 603-8555, Japan; E-Mail: iijima@cc.kyoto-su.ac.jp

2 Department of Animal Medical Sciences, Faculty of Life Sciences, Kyoto Sangyo University, Motoyama, Kamigamo, Kita-Ku, Kyoto 603-8555, Japan; E-Mail: kkonno@cc.kyoto-su.ac.jp

* Author to whom correspondence should be addressed; E-Mail: itanon@cc.kyoto-su.ac.jp; Tel: +81-75-705-3064; Fax: +81-75-705-3064.

Received: 4 July 2011; in revised form: 28 July 2011 / Accepted: 4 August 2011 /

Published: 9 August 2011

\begin{abstract}
Complex interactions between cancer cells and host stromal cells result in the formation of the "tumor microenvironment", where inflammatory alterations involve the infiltration of tumor-associated fibroblasts and inflammatory leukocytes that contribute to the acquisition of malignant characteristics, such as increased cancer cell proliferation, invasiveness, metastasis, angiogenesis, and avoidance of adaptive immunity. The microenvironment of a solid tumor is comprised not only of cellular compartments, but also of bioactive substances, including cytokines, growth factors, and extracellular matrix (ECM). ECM can act as a scaffold for cell migration, a reservoir for cytokines and growth factors, and a signal through receptor binding. During inflammation, ECM components and their degraded fragments act directly and indirectly as inflammatory stimuli in certain cases and regulate the functions of inflammatory and immune cells. One such ECM component, hyaluronan, has recently been implicated to modulate innate immune cell function through pattern recognition toll-like receptors and accelerate the recruitment and activation of tumor-associated macrophages in inflamed cancers. Here, we will summarize the molecular mechanism linking inflammation with ECM remodeling in the tumor microenvironment, with a particular emphasis on the role of hyaluronan in controlling the inflammatory response.
\end{abstract}

Keywords: microenvironment; cancer progression; extracellular matrix; hyaluronan; inflammation 


\section{Introduction}

Cancers develop in a complex and dynamic microenvironment. During cancer initiation and progression, cancer cells receive signals from the tumor microenvironment and communicate bilaterally with host stromal cells [1-4]. These cellular communications extensively alter the cellular and molecular composition of a particular tumor microenvironment. In breast cancer, dynamic changes in the microenvironment are characterized by formation of tumor stroma containing abundant fibrous components as well as by active recruitment of inflammatory cells during the progression from mammary gland hyperplasia to adenoma formation and eventually to cancer [5,6]. It is becoming increasingly clear that the inflammatory changes occurring in the tumor microenvironment are closely linked with the initiation, promotion, and progression of tumorigenesis, and thereby have become the focus of intense research [7,8]. Previous studies have highlighted the important roles of inflammatory cells, especially macrophages, in the development and progression of inflammation-associated cancers [9-11]. Infiltrating pro-inflammatory immune cells within cancers contribute to the maintenance of cytokine-enriched circumstances, which leads to further remodeling of the tumor microenvironment. The first part of this review summarizes our current understanding of the mechanism by which inflammatory reactions alter tumor microenvironments to facilitate malignant transformation and progression. Furthermore, as it has recently become evident that the ECM components and their proteolytic fragments have a considerable impact on the inflammatory response [12], the latter part of this review will focus on the linkage between inflammation and ECM remodeling in the tumor microenvironment.

\section{Inflammatory and Innate Immune Responses in the Tumor Microenvironment, a Brief Overview}

Inflammation is a well established characteristic of cancer. Chronic inflammatory conditions are considered to increase cancer risk and accelerate the development and progression of inflammation-associated cancers. As cancers progress, stromal and inflammatory cells, both of which are recruited into cancer sites, stimulate inflammatory cascades in the tumor microenvironment. Tumor-recruited fibroblasts called tumor-associated fibroblasts (TAFs) are the main cellular components of the stroma of many solid tumors [13,14]. TAFs are known to supply many inflammatory mediators including a variety of cytokines, growth factors, tissue remodeling enzymes such as matrix metalloproteinases (MMPs), and ECM components [15-18], all of which modulate the tumor microenvironment to aid the active mobilization of the inflammatory cells [19-23] (Figure 1). Accumulating evidence indicates that macrophages are important determinants of the inflammatory response in most cancers [9-11]. Studies in op/op mice deficient for the macrophage growth factor CSF-1 have provided strong supporting findings on the close linkage between macrophages and tumor progression [24]. Macrophages are critical effecter cells in the innate immune response, and their lineage comprises a very diverse cell system with respect to phenotypic and functional properties; classical macrophages, termed M1, are capable of efficiently killing tumor cells, while M2 macrophages are immunosuppressive and pro-tumorigenic [9-11]. Tumor-associated macrophages (TAMs), which have little cytotoxicity for tumor cells and can actually promote tumor cell proliferation, resemble M2-polarized macrophages. Recent reports have established that the acquisition of pro-tumorigenic functions by TAMs is driven by various cytokines and signals expressed within the 
tumor microenvironment, thus powering further dynamic change of the tumor microenvironment $[19,21]$. It is therefore believed that TAFs and TAMs play crucial roles in inflammatory alterations of tumor microenvironment in a cooperative fashion (Figure 1).

Figure 1. Schematic of the inflammatory tumor microenvironment. Inflammatory cells produce inflammatory mediators, including cytokines, chemokines, and tissue remodeling enzymes, which in turn amplify and perpetuate the inflammatory cascade. Extracellular matrix (ECM) components serve as a structural scaffold for inflammatory cell infiltration as well as a reservoir for cytokines and growth factors. Enzymatic degradation of ECM components generates bioactive fragments that play key roles in controlling inflammatory processes. Tenascin-C, biglycan, versican, and hyaluronan initiate toll-like receptor (TLR)-mediated innate immune responses.

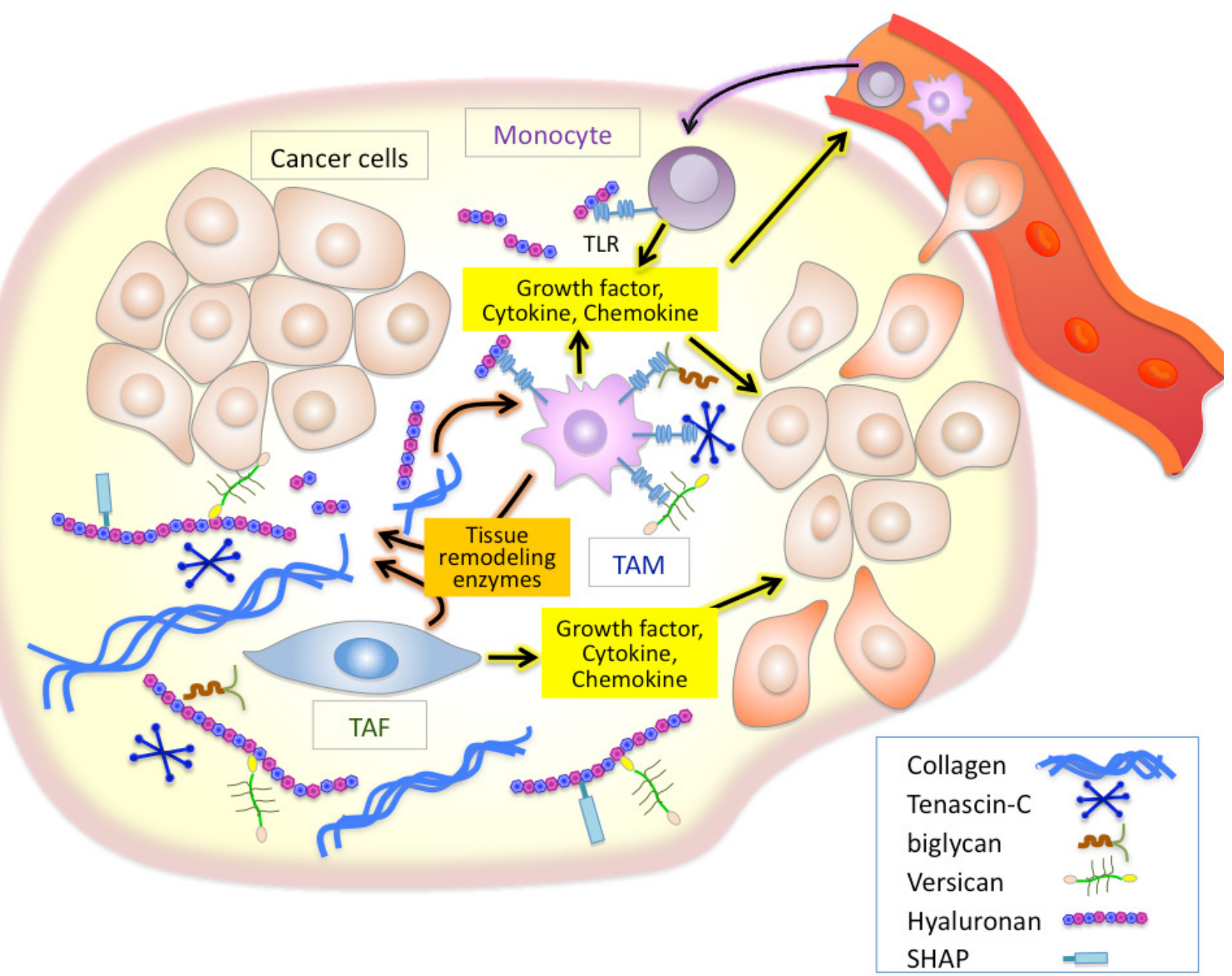

\section{ECM Remodeling in Tumor Microenvironment}

The hallmarks of chronic inflammation include increased infiltration of activated inflammatory cells, mediator release, and turnover of ECM components. An aberrant turnover of ECM components is often detected in cancers, particularly under inflammatory conditions [25,26]. ECM components serve as a structural scaffold for inflammatory cell infiltration as well as a reservoir for cytokines and growth factors [27]. In addition, certain ECM molecules can act indirectly as inflammatory stimuli by 
inducing the expression of proinflammatory genes. The degradation of ECM components gives rise to the generation of bioactive fragments that play key roles in controlling numerous events, including tissue remodeling, inflammation, angiogenesis, tumor growth, and metastasis [28]. Matrikines, which are fragments obtained by proteolytic cleavage of ECM constituents, have biological functions distinct from those of parental proteins and act directly as inflammatory stimuli in certain cases [29,30]. Proteolytic fragments generated from collagen types I and IV, fibronectin, laminins, elastin, entactin/nidogen, and thrombospondin-1/2 have been shown to act as chemoattractants for inflammatory cells. In addition to the direct action of these degraded fragments, indirect effects on inflammatory responses are also seen via specific protease and cytokine gene induction that are necessary for matrix remodeling and inflammation.

There is growing evidence that ECM molecules can activate the innate immune system as endogenous antigens in a manner analogous to pathogen-associated molecular patterns [31-33]. Of such ECM molecules, tenascin-C, biglycan, versican, and hyaluronan have been demonstrated to initiate toll-like receptor (TLR)-mediated innate immune responses [34-38] (Figure 1). Tenascin-C is a proinflammatory extracellular glycoprotein whose expression is specifically and rapidly induced in response to tissue injury [39]. Tenascin-C-mediated chronic inflammation is clearly associated with the symptoms of rheumatoid arthritis [39]. One study also suggested that tenascin-C induces cytokine synthesis in macrophages and synovial fibroblasts as an endogenous activator of TLR4 in arthritic joint disease [34]. Since elevated tenascin-C expression is often observed in chronic inflammation of tumor stroma, an analogous mechanism may play an important role in inflammation in the tumor microenvironment [39]. Biglycan is a small leucine-rich proteoglycan with a core protein and one or more chondroitin/dermatan sulfate chains. Recently, biglycan has been reported to bind to the endocytic mannose receptor, a pattern recognition receptor expressed on macrophages and dendritic cells (DCs) [40]. Binding of biglycan significantly increases IL-10 production and decreases IL-12 in lipopolysaccharide-maturing DCs, suggesting a down-regulation of Th1-polarized immune responses. Schaefer et al. has demonstrated that biglycan activates intracellular signaling pathways through TLR2 and TLR4 in macrophages, eventually leading to an enhanced synthesis of proinflammatory TNF- $\alpha$ and MIP-2 [35].

Versican, a large chondroitin sulfate proteoglycan, is structurally composed of a hyaluronan-binding G1 domain at the $N$-terminus, glycosaminoglycan attachment regions, and a C-terminal G3 domain containing two epidermal growth factor (EGF)-like repeats, a C-type lectin-like motif, and a complement binding protein (CBP)-like motif [41] (Figure 2). Versican is involved in ECM assembly via the association of these specific domains with various other ECM constituents, such as hyaluronan, tenascin-C, fibronectin, fibulin-1, and fibrillin. Through interactions with ECM molecules, versican regulates many cellular processes of adhesion, migration, proliferation, apoptosis, and angiogenesis, all events implicated in tumor progression and metastasis. Elevated expression of versican both in tumor stroma and in cancer cells is frequently associated with poor outcome in breast, brain, ovary, and prostate cancers, sarcoma, and mesothelioma [42]. Due to its interactions with CXC and CC chemokines, versican may also act as a chemokine reservoir and facilitate the recruitment of inflammatory cells via chemokine release [43]. 
Figure 2. Schematic of the hyaluronan-enriched extracellular matrix. Hyaluronan assembles ECM through association with its binding partners, such as versican proteoglycan and SHAP, and serves as a structural scaffold for inflammatory cells.

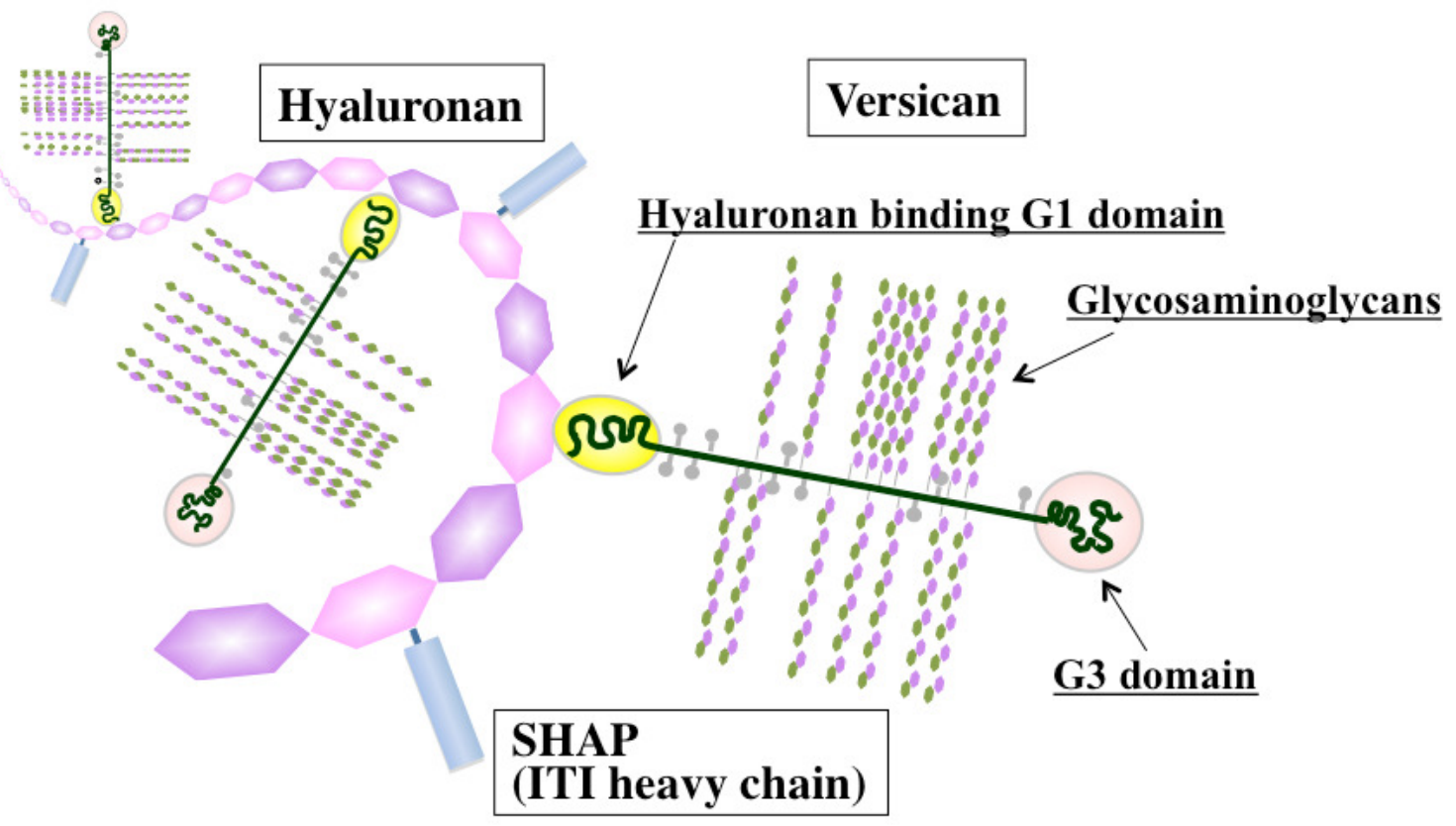

Another suspected role of versican lies in its ability to mediate the retention of inflammatory leukocytes through interactions with cell surface CD44 and L-selectin receptors [44]. Weight and colleagues have reported an important role for versican in the hyaluronan-dependent binding of monocytes to the ECM of lung fibroblasts stimulated with poly I:C, although the responsible receptor has not been identified yet [45]. By revealing the molecular cues that promote cancer metastasis, Karin et al. recently uncovered a new inflammatory pathway in which versican activates macrophages via TLR2 and its co-receptors TLR6 and CD14 [36]. In that study, they further showed that ligation of TLR2 by versican elicits the production of proinflammatory cytokines, providing a positive link between inflammation and cancer metastasis.

Hyaluronan serves as an attachment ligand for receptors on inflammatory cells and is partly responsible for leukocyte retention in the inflammatory response [46]. CD44 is one such receptor and has been shown to participate in monocyte/macrophage retention and activation in inflammatory sites [47] (Figure 3). Similarly to other ECM molecules, hyaluronan degradation products have the ability to induce specific gene expression programs for proteases and cytokines that are necessary for inflammation and matrix remodeling. Several recent studies have shown that hyaluronan fragments activate innate immune responses by interacting with TLR2 and TLR4 and inducing inflammatory gene expression in a variety of immune cells [37,38,48] (Figure 3). In light of this, we will focus the latter half of this review on the key role of hyaluronan in facilitating the inflammatory circuit in the tumor microenvironment. 
Figure 3. Inflammatory responses of monocytes/macrophages to hyaluronan. During tumor progression, high molecular-weight (HMW) hyaluronan forms part of the tumor microenvironment by linking its binding partners into macromolecular aggregates. The hyaluronan-enriched tumor microenvironment accelerates recruitment and activation of monocytes/macrophages. Low molecular-weight (LMW) hyaluronan generated by hyaluronan fragmentation accelerates inflammatory responses via TLRs on macrophages and regulates inflammatory gene expression.

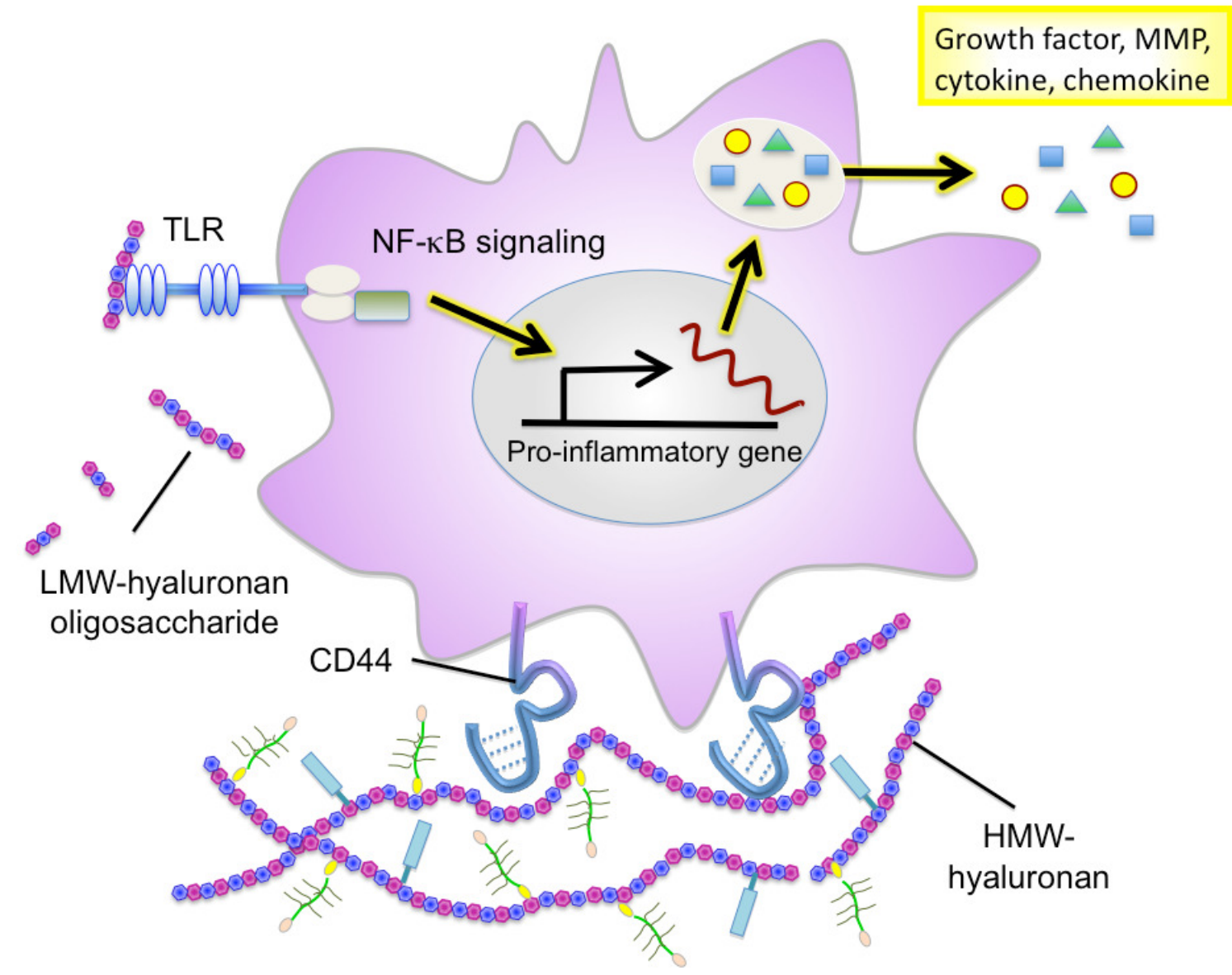

\section{Hyaluronan Accumulation in Cancers}

Hyaluronan is the main component of the tumor microenvironment and has thus become an increasingly important target for cancer therapy [49]. While hyaluronan is essential for the construction of normal tissue architecture, it is also known as a polysaccharide closely related to cancer [50]. The association between a rise in hyaluronan and cancer dates back to the 1950s, when a study first reported increased hyaluronan production in a mesothelioma [51,52]. Since then, hyaluronan has been accepted as a significant prognostic factor in many types of cancers, and high levels of this molecule are correlated with a poor prognosis [53,54]; in several studies, the proportion of hyaluronan-positive cells and intensity of hyaluronan staining in breast cancer tissues were closely correlated with a poor survival rate and prognosis [55]. In colorectal cancers, the 5-year survival rate of patients with a large fraction of hyaluronan-positive cancer cells was reported to be only $20 \%$ [56]. Poorly differentiated or high-grade tumors generally have more stromal hyaluronan than well-differentiated tumors, and multivariate analyses have confirmed the rate of positive staining for 
hyaluronan in tumor stroma to be an independent poor prognostic factor. In ovarian cancers, the hyaluronan level in tumor stroma was correlated with the degree of cancer cell undifferentiation and cancer stage [57]. Therefore, hyaluronan, and especially stromal hyaluronan, may be an important factor determining the malignant characteristics of cancers.

\section{Biosynthesis, Catabolism and Physiopathological Functions of Hyaluronan}

Hyaluronan is a simple polysaccharide molecule composed of repeating disaccharide units of alternating $\mathrm{N}$-acetylglucosamine and glucuronic acid (Figure 4). The dynamic turnover of hyaluronan is tightly balanced by its synthesis and degradation to maintain a specific concentration and chain length in tissues [58]. Hyaluronan synthesis is catalyzed by one of three isoforms of the HAS enzyme: HAS1, HAS2, and HAS3. Structurally, all HAS enzymes are integral membrane proteins composed of multiple membrane-spanning regions with hydrophobic amino acid clusters and a large cytoplasmic loop. In this loop, there are two catalytic sites participating in the transfer of UDP-GlcNAc and UDP-GlcA substrates. These isoforms however differ from one another in the length of sugar chains synthesized and ECM-forming ability [59]. Regarding the subcellular localization of HAS enzymes, a recent study has also proposed that latent enzymes reside around the endoplasmic reticulum (ER) and nuclear membrane and synthesize cable-like hyaluronan structures upon particular cellular stresses, such as ER stress [60]. This provides evidence for multiple regulatory mechanisms of HA biosynthesis, where structurally different HA molecules are synthesized in different subcellular compartments depending on cellular state.

Figure 4. Hyaluronan structure and degradation. Hyaluronan is composed of repeating disaccharide units of $N$-acetylglucosamine (GlcNAc) $\beta(1 \rightarrow 4)$-glucuronic acid (GlcA) $\beta(1 \rightarrow 3)$. Hyaluronidase cleaves $N$-acetylglucosamine $\beta(1 \rightarrow 4)$ glycoside bonds in hyaluronan.

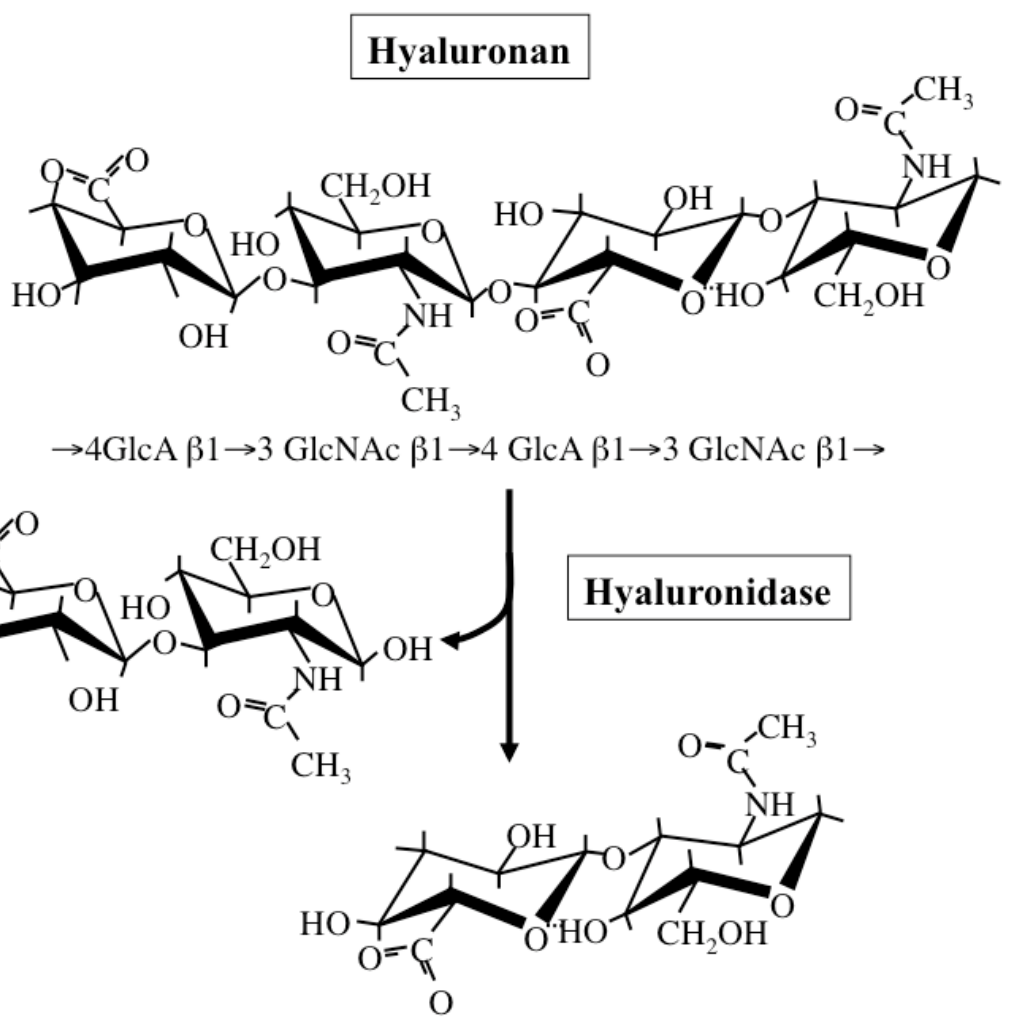


Hyaluronan catabolism is predominantly regulated by several hyaluronidases (Figure 4), which are classified as endo- $\beta-N$-acetylglucosaminidases according to their hydrolytic mechanisms [61]. Six structurally homologous hyaluronidase-related genes (HYAL-1, -2, -3, -4, -P1, and PH-20) have been identified so far in mammals [61]. Among them, the main tissue hyaluronidases HYAL-1 and -2 regulate hyaluronan degradation in a sequential series of catabolic reactions. HYAL-2 degrades high molecular-weight hyaluronan into fragments of up to $20 \mathrm{kDa}$, HYAL-1 breaks down hyaluronan into fragments of up to $800 \mathrm{Da}$. Since gene expression of HYAL-1 and -2 is up-regulated under pathological conditions, hyaluronidase-mediated hyaluronan degradation appears to occur in subjects with cancer and chronic inflammation.

The physiopathological functions of hyaluronan vary in a manner dependent on its sugar chain length [62]. High molecular-weight (HMW) hyaluronan assembles the ECM in association with binding molecules (Figure 3). This type of hyaluronan ECM is believed to maintain tissue structure and serve as a scaffold for cell adhesion and migration. HMW hyaluronan exhibits space-filling and water-retention properties and functions to modulate the pericellular ECM microenvironment. Alternatively, oligosaccharides produced from the catabolic activity of hyaluronidase diffuse through tissues and bind to hyaluronan receptors on adjacent cells, thereby acting as intracellular signals such as NF-kB and Erk [63]. The promotion of tumor angiogenesis and induction of inflammation by hyaluronan oligosaccharides are well defined, as shown in Table 1.

Table 1. Inflammatory actions of hyaluronan and its oligosaccharides.

\begin{tabular}{lll}
\hline $\begin{array}{l}\text { Hyaluronan length } \\
\text { (mers or kDa) }\end{array}$ & Inflammatory-related functions of hyaluronan & References \\
\hline $4-6$ mers & Activation of NF-KB and upregulation of MMP expression & {$[64,65]$} \\
$4-16$ mers & $\begin{array}{l}\text { Induction of cytokines and chemokines } \\
\text { Activation and maturation of DCs }\end{array}$ & {$[37,66-68]$} \\
$6-32$ mers & $\begin{array}{l}\text { Stimulation of angiogenesis } \\
\text { Induction of proinflammatory cytokines and chemokines } \\
\text { in macrophage }\end{array}$ & {$[69-71]$} \\
& $\begin{array}{l}\text { Induction of iNOS and COX-2 in macrophage } \\
\text { Activation of innate immunity }\end{array}$ & {$[48,72-77]$} \\
\hline
\end{tabular}

MMP: matrix metalloprotease; DC: dendritic cell; EC: endothelial cell.

\section{Aberrant Hyaluronan Turnover and Function in Cancers}

The rates of hyaluronan synthesis and degradation are much higher in cancers than in normal tissues [50]. The malignant transformation of cells frequently impairs regulation of hyaluronan synthesis and leads to excessive hyaluronan production [53,78]. Studies using several cancer cell lines reported that HAS expression was increased in cancer cells whose ability to produce hyaluronan was enhanced [79,80]. Furthermore, amplification of the genomic region that includes the HAS2 gene has also been found with high frequency in prostate cancer [81]. Although not directly proven, HAS gene amplification may be directly linked to increased hyaluronan synthesis in cancer cells. These results suggest that abnormal hyaluronan accumulation in cancer tissue is the result of transformed cells acquiring a high hyaluronan-producing ability associated with increased HAS expression. In addition 
to excess hyaluronan production in cancer cells, TAFs and other stromal cells are also considered to contribute significantly to the accumulation of hyaluronan [82]. In cancer tissues, gene expression of hyaluronan synthases in cancer cells and fibroblasts is elevated following exposure to various growth factors and cytokines, resulting in a marked increase in hyaluronan synthesis [82-84]. Pathological analysis of clinical samples has demonstrated that the increased expression of HAS1 among the three HAS isoforms in human colorectal cancer tissues is correlated with lymph node metastasis [85]. Although a close correlation between HAS gene expression and tumor formation and metastasis has been suggested by many studies using cultured tumor cells [86,87], exceptions have also been observed. Enegd et al. reported that the forced expression of HAS2 in glioma cells resulted in the suppression of tumor formation, and postulated that a balance between hyaluronan degradation by hyaluronidase and synthesis by HAS was responsible for tumor formation [88]. These observations therefore suggest that hyaluronan promotes tumor progression at a specific concentration range and sugar-chain length, but inhibits tumor formation at other concentrations or sugar-chain lengths.

The expression of such hyaluronidases is up-regulated in certain cancers [89-91]. Pathological analysis of clinical samples has shown that HYAL-1 expression is a good prognostic indicator of prostate and bladder cancers [89], while the analysis of breast cancer cell lines has revealed that highly invasive cancer cells express high levels of HYAL-2 [92]. The association between hyaluronidase and tumor formation has been directly confirmed by the fact that HYAL-2 overexpression in mouse astrocytoma promotes the formation of tumors [93]. On the other hand, similarly to HAS, hyaluronidase exhibited a completely opposite effect in some cases; a human breast cancer transplanted into immunodeficient (SCID) mice markedly regressed after intravenous administration of testicular hyaluronidase [94], and HYAL-1 overexpression in a colorectal cancer model inhibited tumor formation [95]. These conflicting results support the notion of a delicate balance between hyaluronan degradation and synthesis in tumor development.

In advanced cancer, therefore, aberrant synthesis and degradation of hyaluronan result in the formation of an extremely unusual microenvironment characterized by the accumulation of HMW hyaluronan and excessive hyaluronan oligosaccharides, which may facilitate the malignant transformation and survival of cancer cells.

\section{The Roles of Hyaluronan in Inflammation}

Recent studies have firmly implicated hyaluronan in the regulation of immune and inflammatory cell responses. Hyaluronan synthesis is regulated by numerous pro-inflammatory factors and cytokines. Hyaluronan oligosaccharides act on monocytes/macrophages to differentiate them into M2 macrophages [96], suggesting that they can regulate polarization of myeloid lineages by affecting the balance of Th1/Th2 cytokines in local environments. Emerging evidence further points to an important role of hyaluronan fragments in the induction of many inflammatory cytokines (IL-8, IL-12, and TNF- $\alpha$ ) and chemokines (MIP-1 $\alpha$, MIP-1 $\beta$, KC, RANTES, MCP-1, and IFN-inducible protein-10) in a variety of immune cells [66,72,73] (Figure 3). Furthermore, hyaluronan fragments serve as an endogenous ligand for TLRs, which is crucial for the activation of innate immune cells $[37,38,48]$. Interestingly, TLR-mediated activation is hyaluronan size-dependent and is only seen in the presence of smaller molecule fragments 4-16 oligosaccharides in size. 
Considerable information has been accumulated on the ability of hyaluronan-associated molecules to modulate hyaluronan functions affecting immune and inflammatory responses (Figure 2). de la Motte et al. showed that hyaluronan and its associated molecules formed cable-like ECM structures that were involved in the adhesion and recruitment of monocytes through association with hyaluronan receptor CD44 [97]. Several hyaluronan-binding partners, such as SHAP, tumor necrosis factor stimulated gene 6 (TSG-6), and versican, are located along the strands of cable-like ECM containing hyaluronan and are also expected to play crucial roles in structure formation [98]. SHAP, a serum-derived hyaluronan-associated protein, participates in the construction of such cable-like structures and enhances hyaluronan-mediated leukocyte adhesion after forming a complex with hyaluronan [99] (Figure 2). SHAP corresponds to the heavy chains of the plasma inter- $\alpha$-trypsin inhibitor (ITI) family and covalently binds to hyaluronan via a unique ester bond. The transfer of SHAP to hyaluronan is mediated by TSG-6 through a hyaluronan-binding link module [100]. Serum levels of SHAP-hyaluronan complexes have been reported to be closely associated with the patho-physiology of inflammatory diseases, such as rheumatoid arthritis, and correlated with the clinical stages of chronic hepatitis [101,102]. A similar observation was also made in ovarian cancers, where SHAP serum levels were correlated with clinical outcome [103]. Since hyaluronan and versican can stimulate macrophage cytokine and chemokine production in a TLR-dependent manner, the co-localization of these macromolecules may imply their cooperative action in the innate immune response. Our recent study demonstrated the preferential engagement of immunosuppressive M2 macrophages in a hyaluronan- and versican-rich stromal microenvironment [104]. The effects were typically observed only with the hyaluronan-versican complex, supporting their cooperative action as modulators of innate immunity. Taken together, these observations indicate that the hyaluronan-enriched tumor microenvironment regulates innate immune and inflammatory responses by enhancing the recruitment of innate immune cells and modulating inflammatory gene expression.

\section{Conclusions and Future Prospects}

Recent findings have shed new light on the inflammatory alterations that take place in the tumor microenvironment. Emerging evidence supports the view that ECM is not only remodeled as a result of inflammation, but also directly and indirectly influences the inflammation circuit. During inflammation, ECM components and their fragments play substantial roles in inflammation cascades; matrikines derived proteolytically from ECM components act directly as inflammatory stimuli. Tenascin-C, proteoglycans, and hyaluronan initiate TLR-mediated inflammation reactions. These components also have important functions as reservoirs for inflammatory cytokines, inducers of gene expression, and modulators of biological activity. It therefore appears that the enrichment of these ECM molecules in the tumor microenvironment is a sign of inflammation. Since the inflammatory matrix provides an environment amenable to the acquisition of malignant characteristics for tumor cells, further elucidation of the precise mechanisms controlling inflammatory matrix reconstruction will allow us to develop novel and promising therapeutic strategies to prevent the aberrant matrix turnover that is associated with cancer cell growth, invasion, metastasis, and angiogenesis. 


\section{Acknowledgements}

This work was supported in part by KAKENHI (grants-in-aid for scientific research) from the Ministry of Education, Culture, Sports, Science and Technology of Japan and a grant from Mizutani Foundation for Glycoscience (N. Itano).

\section{References}

1. Allen, M.; Jones, L.J. Jekyll and Hyde: the role of the microenvironment on the progression of cancer. J. Pathol. 2011, 223, 162-176.

2. Joyce, J.A. Therapeutic targeting of the tumor microenvironment. Cancer Cell 2005, 7, 513-520.

3. Mueller, M.M.; Fusenig, N.E. Friends or foes-bipolar effects of the tumour stroma in cancer. Nat. Rev. Cancer 2004, 4, 839-849.

4. Bhowmick, N.A.; Moses, H.L. Tumor-stroma interactions. Curr. Opin. Genet. Dev. 2005, 15, 97-101.

5. Wiseman, B.S.; Werb, Z. Stromal effects on mammary gland development and breast cancer. Science 2002, 296, 1046-1049.

6. DeNardo, D.G.; Coussens, L.M. Inflammation and breast cancer. Balancing immune response: crosstalk between adaptive and innate immune cells during breast cancer progression. Breast Cancer Res. 2007, 9, 212.

7. Lin, W.W.; Karin, M. A cytokine-mediated link between innate immunity, inflammation, and cancer. J. Clin. Invest. 2007, 117, 1175-1183.

8. Allavena, P.; Garlanda, C.; Borrello, M.G.; Sica, A.; Mantovani, A. Pathways connecting inflammation and cancer. Curr. Opin. Genet. Dev. 2008, 18, 3-10.

9. Mantovani, A.; Schioppa, T.; Porta, C.; Allavena, P.; Sica, A. Role of tumor-associated macrophages in tumor progression and invasion. Cancer Metast. Rev. 2006, 25, 315-322.

10. Lewis, C.E.; Pollard, J.W. Distinct role of macrophages in different tumor microenvironments. Cancer Res. 2006, 66, 605-612.

11. Sica, A.; Larghi, P.; Mancino, A.; Rubino, L.; Porta, C.; Totaro, M.G.; Rimoldi, M.; Biswas, S.K.; Allavena, P.; Mantovani, A. Macrophage polarization in tumour progression. Semin. Cancer Biol. 2008, 18, 349-355.

12. Chiodoni, C.; Colombo, M.P.; Sangaletti, S. Matricellular proteins: from homeostasis to inflammation, cancer, and metastasis. Cancer Metast. Rev. 2010, 29, 295-307.

13. Kalluri, R.; Zeisberg, M. Fibroblasts in cancer. Nat. Rev. Cancer 2006, 6, 392-401.

14. Yazhou, C.; Wenlv, S.; Weidong, Z.; Licun, W. Clinicopathological significance of stromal myofibroblasts in invasive ductal carcinoma of the breast. Tumour Biol. 2004, 25, 290-295.

15. Desmouliere, A.; Guyot, C.; Gabbiani, G. The stroma reaction myofibroblast: a key player in the control of tumor cell behavior. Int. J. Dev. Biol. 2004, 48, 509-517.

16. Dong, J.; Grunstein, J.; Tejada, M.; Peale, F.; Frantz, G.; Liang, W.C.; Bai, W.; Yu, L.; Kowalski, J.; Liang, X.; Fuh, G.; Gerber, H.P.; Ferrara, N. VEGF-null cells require PDGFR alpha signaling-mediated stromal fibroblast recruitment for tumorigenesis. EMBO J. 2004, 23, 2800-2810. 
17. Orimo, A.; Gupta, P.B.; Sgroi, D.C.; Arenzana-Seisdedos, F.; Delaunay, T.; Naeem, R.; Carey, V.J.; Richardson, A.L.; Weinberg, R.A. Stromal fibroblasts present in invasive human breast carcinomas promote tumor growth and angiogenesis through elevated SDF-1/CXCL12 secretion. Cell 2005, 121, 335-348.

18. Kessenbrock, K.; Plaks, V.; Werb, Z. Matrix metalloproteinases: regulators of the tumor microenvironment. Cell 2010, 141, 52-67.

19. Schoppmann, S.F.; Birner, P.; Stockl, J.; Kalt, R.; Ullrich, R.; Caucig, C.; Kriehuber, E.; Nagy, K.; Alitalo, K.; Kerjaschki, D. Tumor-associated macrophages express lymphatic endothelial growth factors and are related to peritumoral lymphangiogenesis. Am. J. Pathol. 2002, 161, 947-956.

20. Robinson, S.C.; Coussens, L.M. Soluble mediators of inflammation during tumor development. Adv. Cancer Res. 2005, 93, 159-187.

21. Chitu, V.; Stanley, E.R. Colony-stimulating factor-1 in immunity and inflammation. Curr. Opin. Immunol. 2006, 18, 39-48.

22. Chung, A.S.; Kao, W.J. Fibroblasts regulate monocyte response to ECM-derived matrix: the effects on monocyte adhesion and the production of inflammatory, matrix remodeling, and growth factor proteins. J. Biomed. Mater. Res. A 2009, 89, 841-853.

23. Zhang, J.; Chen, L.; Xiao, M.; Wang, C.; Qin, Z. FSP1 ${ }^{+}$fibroblasts promote skin carcinogenesis by maintaining MCP-1-mediated macrophage infiltration and chronic inflammation. Am. J. Pathol. 2011, 178, 382-390.

24. Nowicki, A.; Szenajch, J.; Ostrowska, G.; Wojtowicz, A.; Wojtowicz, K.; Kruszewski, A.A.; Maruszynski, M.; Aukerman, S.L.; Wiktor-Jedrzejczak, W. Impaired tumor growth in colony-stimulating factor 1 (CSF-1)-deficient, macrophage-deficient op/op mouse: evidence for a role of CSF-1-dependent macrophages in formation of tumor stroma. Int. J. Cancer 1996, 65, 112-119.

25. Pupa, S.M.; Menard, S.; Forti, S.; Tagliabue, E. New insights into the role of extracellular matrix during tumor onset and progression. J. Cell Physiol. 2002, 192, 259-267.

26. Jodele, S.; Blavier, L.; Yoon, J.M.; DeClerck, Y.A. Modifying the soil to affect the seed: Role of stromal-derived matrix metalloproteinases in cancer progression. Cancer Metast. Rev. 2006, 25, $35-43$.

27. Sorokin, L. The impact of the extracellular matrix on inflammation. Nat. Rev. Immunol. 2010, 10, 712-723.

28. Adair-Kirk, T.L.; Senior, R.M. Fragments of extracellular matrix as mediators of inflammation. Int. J. Biochem. Cell Biol. 2008, 40, 1101-1110.

29. Tran, K.T.; Lamb, P.; Deng, J.S. Matrikines and matricryptins: Implications for cutaneous cancers and skin repair. J. Dermatol. Sci. 2005, 40, 11-20.

30. Duca, L.; Floquet, N.; Alix, A.J.; Haye, B.; Debelle, L. Elastin as a matrikine. Crit. Rev. Oncol. Hematol. 2004, 49, 235-244.

31. Schaefer, L. Extracellular matrix molecules: endogenous danger signals as new drug targets in kidney diseases. Curr. Opin. Pharmacol. 2010, 10, 185-190.

32. Iozzo, R.V.; Schaefer, L. Proteoglycans in health and disease: Novel regulatory signaling mechanisms evoked by the small leucine-rich proteoglycans. FEBS J. 2010, 277, 3864-3875. 
33. Jiang, D.; Liang, J.; Noble, P.W. Hyaluronan as an immune regulator in human diseases. Physiol. Rev. 2011, 91, 221-264.

34. Midwood, K.; Sacre, S.; Piccinini, A.M.; Inglis, J.; Trebaul, A.; Chan, E.; Drexler, S.; Sofat, N.; Kashiwagi, M.; Orend, G.; Brennan, F.; Foxwell, B. Tenascin-C is an endogenous activator of Toll-like receptor 4 that is essential for maintaining inflammation in arthritic joint disease. Nat. Med. 2009, 15, 774-780.

35. Schaefer, L.; Babelova, A.; Kiss, E.; Hausser, H.J.; Baliova, M.; Krzyzankova, M.; Marsche, G.; Young, M.F.; Mihalik, D.; Gotte, M.; Malle, E.; Schaefer, R.M.; Grone, H.J. The matrix component biglycan is proinflammatory and signals through Toll-like receptors 4 and 2 in macrophages. J. Clin. Invest. 2005, 115, 2223-2233.

36. Kim, S.; Takahashi, H.; Lin, W.W.; Descargues, P.; Grivennikov, S.; Kim, Y.; Luo, J.L.; Karin, M. Carcinoma-produced factors activate myeloid cells through TLR2 to stimulate metastasis. Nature 2009, 457, 102-106.

37. Termeer, C.; Benedix, F.; Sleeman, J.; Fieber, C.; Voith, U.; Ahrens, T.; Miyake, K.; Freudenberg, M.; Galanos, C.; Simon, J.C. Oligosaccharides of Hyaluronan activate dendritic cells via toll-like receptor 4. J. Exp. Med. 2002, 195, 99-111.

38. Jiang, D.; Liang, J.; Fan, J.; Yu, S.; Chen, S.; Luo, Y.; Prestwich, G.D.; Mascarenhas, M.M.; Garg, H.G.; Quinn, D.A.; Homer, R.J.; Goldstein, D.R.; Bucala, R.; Lee, P.J.; Medzhitov, R.; Noble, P.W. Regulation of lung injury and repair by Toll-like receptors and hyaluronan. Nat. Med. 2005, 11, 1173-1179.

39. Chiquet-Ehrismann, R.; Chiquet, M. Tenascins: Regulation and putative functions during pathological stress. J. Pathol. 2003, 200, 488-499.

40. Chieppa, M.; Bianchi, G.; Doni, A.; Del Prete, A.; Sironi, M.; Laskarin, G.; Monti, P.; Piemonti, L.; Biondi, A.; Mantovani, A.; Introna, M.; Allavena, P. Cross-linking of the mannose receptor on monocyte-derived dendritic cells activates an anti-inflammatory immunosuppressive program. J. Immunol. 2003, 171, 4552-4560.

41. Wight, T.N. Versican: A versatile extracellular matrix proteoglycan in cell biology. Curr. Opin. Cell Biol. 2002, 14, 617-623.

42. Theocharis, A.D.; Skandalis, S.S.; Tzanakakis, G.N.; Karamanos, N.K. Proteoglycans in health and disease: Novel roles for proteoglycans in malignancy and their pharmacological targeting. Febs. J. 2010, 277, 3904-3923.

43. Hirose, J.; Kawashima, H.; Yoshie, O.; Tashiro, K.; Miyasaka, M. Versican interacts with chemokines and modulates cellular responses. J. Biol. Chem. 2001, 276, 5228-5234.

44. Wu, Y.J.; La Pierre, D.P.; Wu, J.; Yee, A.J.; Yang, B.B. The interaction of versican with its binding partners. Cell Res. 2005, 15, 483-494.

45. Potter-Perigo, S.; Johnson, P.Y.; Evanko, S.P.; Chan, C.K.; Braun, K.R.; Wilkinson, T.S.; Altman, L.C.; Wight, T.N. Polyinosine-polycytidylic acid stimulates versican accumulation in the extracellular matrix promoting monocyte adhesion. Am. J. Respir. Cell Mol. Biol. 2010, 43, 109-120.

46. Termeer, C.; Sleeman, J.P.; Simon, J.C. Hyaluronan-Magic glue for the regulation of the immune response? Trends Immunol. 2003, 24, 112-114.

47. Lesley, J.; Hyman, R.; English, N.; Catterall, J.B.; Turner, G.A. CD44 in inflammation and metastasis. Glycoconj. J. 1997, 14, 611-622. 
48. Scheibner, K.A.; Lutz, M.A.; Boodoo, S.; Fenton, M.J.; Powell, J.D.; Horton, M.R. Hyaluronan fragments act as an endogenous danger signal by engaging TLR2. J. Immunol. 2006, 177, 1272-1281.

49. Itano, N.; Zhuo, L.; Kimata, K. Impact of the hyaluronan-rich tumor microenvironment on cancer initiation and progression. Cancer Sci. 2008, 99, 1720-1725.

50. Toole, B.P. Hyaluronan: From extracellular glue to pericellular cue. Nat. Rev. Cancer 2004, 4, 528-539.

51. Blix, G. Hyaluronic acid in the pleural and peritoneal fluids from a case of mesothelioma. Acta Soc. Med. Ups. 1951, 56, 47-50.

52. Truedsson, E. A case of mesothelioma of the pleura and peritoneum producing hyaluronic acid. Acta Soc. Med. Ups. 1951, 56, 39-45.

53. Hopwood, J.J.; Dorfman, A. Glycosaminoglycan synthesis by cultured human skin fibroblasts after transformation with simian virus 40. J. Biol. Chem. 1977, 252, 4777-4785.

54. Delpech, B.; Chevallier, B.; Reinhardt, N.; Julien, J.P.; Duval, C.; Maingonnat, C.; Bastit, P.; Asselain, B. Serum hyaluronan (hyaluronic acid) in breast cancer patients. Int. J. Cancer 1990, 46, 388-390.

55. Auvinen, P.; Tammi, R.; Parkkinen, J.; Tammi, M.; Agren, U.; Johansson, R.; Hirvikoski, P.; Eskelinen, M.; Kosma, V.M. Hyaluronan in peritumoral stroma and malignant cells associates with breast cancer spreading and predicts survival. Am. J. Pathol. 2000, 156, 529-536.

56. Ropponen, K.; Tammi, M.; Parkkinen, J.; Eskelinen, M.; Tammi, R.; Lipponen, P.; Agren, U.; Alhava, E.; Kosma, V.M. Tumor cell-associated hyaluronan as an unfavorable prognostic factor in colorectal cancer. Cancer Res. 1998, 58, 342-347.

57. Anttila, M.A.; Tammi, R.H.; Tammi, M.I.; Syrjanen, K.J.; Saarikoski, S.V.; Kosma, V.M. High levels of stromal hyaluronan predict poor disease outcome in epithelial ovarian cancer. Cancer Res. 2000, 60, 150-155.

58. Itano, N. Simple primary structure, complex turnover regulation and multiple roles of hyaluronan. J. Biochem. 2008, 144, 131-137.

59. Itano, N.; Sawai, T.; Yoshida, M.; Lenas, P.; Yamada, Y.; Imagawa, M.; Shinomura, T.; Hamaguchi, M.; Yoshida, Y.; Ohnuki, Y.; Miyauchi, S.; Spicer, A.P.; McDonald, J.A.; Kimata, K. Three isoforms of mammalian hyaluronan synthases have distinct enzymatic properties. J. Biol. Chem. 1999, 274, 25085-25092.

60. Day, A.J.; de la Motte, C.A. Hyaluronan cross-linking: a protective mechanism in inflammation? Trends Immunol. 2005, 26, 637-643.

61. Csoka, A.B.; Frost, G.I.; Stern, R. The six hyaluronidase-like genes in the human and mouse genomes. Matrix Biol. 2001, 20, 499-508.

62. Laurent, T.C.; Fraser, J.R. Hyaluronan. FASEB J. 1992, 6, 2397-2404.

63. Stern, R.; Asari, A.A.; Sugahara, K.N. Hyaluronan fragments: an information-rich system. Eur. J. Cell Biol. 2006, 85, 699-715.

64. Fieber, C.; Baumann, P.; Vallon, R.; Termeer, C.; Simon, J.C.; Hofmann, M.; Angel, P.; Herrlich, P.; Sleeman, J.P. Hyaluronan-oligosaccharide-induced transcription of metalloproteases. J. Cell Sci. 2004, 117, 359-367. 
65. Voelcker, V.; Gebhardt, C.; Averbeck, M.; Saalbach, A.; Wolf, V.; Weih, F.; Sleeman, J.; Anderegg, U.; Simon, J. Hyaluronan fragments induce cytokine and metalloprotease upregulation in human melanoma cells in part by signalling via TLR4. Exp. Dermatol. 2008, 17, 100-107.

66. McKee, C.M.; Penno, M.B.; Cowman, M.; Burdick, M.D.; Strieter, R.M.; Bao, C.; Noble, P.W. Hyaluronan (HA) fragments induce chemokine gene expression in alveolar macrophages. The role of HA size and CD44. J. Clin. Invest. 1996, 98, 2403-2413.

67. Taylor, K.R.; Trowbridge, J.M.; Rudisill, J.A.; Termeer, C.C.; Simon, J.C.; Gallo, R.L. Hyaluronan fragments stimulate endothelial recognition of injury through TLR4. J. Biol. Chem. 2004, 279, 17079-17084.

68. Takahashi, Y.; Li, L.; Kamiryo, M.; Asteriou, T.; Moustakas, A.; Yamashita, H.; Heldin, P. Hyaluronan fragments induce endothelial cell differentiation in a CD44- and CXCL1/GRO1-dependent manner. J. Biol. Chem. 2005, 280, 24195-24204.

69. West, D.C.; Hampson, I.N.; Arnold, F.; Kumar, S. Angiogenesis induced by degradation products of hyaluronic acid. Science 1985, 228, 1324-1326.

70. Sattar, A.; Rooney, P.; Kumar, S.; Pye, D.; West, D.C.; Scott, I.; Ledger, P. Application of angiogenic oligosaccharides of hyaluronan increases blood vessel numbers in rat skin. J. Invest. Dermatol. 1994, 103, 576-579.

71. Montesano, R.; Kumar, S.; Orci, L.; Pepper, M.S. Synergistic effect of hyaluronan oligosaccharides and vascular endothelial growth factor on angiogenesis in vitro. Lab. Invest. 1996, 75, 249-262.

72. Hodge-Dufour, J.; Noble, P.W.; Horton, M.R.; Bao, C.; Wysoka, M.; Burdick, M.D.; Strieter, R.M.; Trinchieri, G.; Pure, E. Induction of IL-12 and chemokines by hyaluronan requires adhesion-dependent priming of resident but not elicited macrophages. J. Immunol. 1997, 159, 2492-2500.

73. Horton, M.R.; Burdick, M.D.; Strieter, R.M.; Bao, C.; Noble, P.W. Regulation of hyaluronan-induced chemokine gene expression by IL-10 and IFN-gamma in mouse macrophages. J. Immunol. 1998, 160, 3023-3030.

74. Noble, P.W.; McKee, C.M.; Cowman, M.; Shin, H.S. Hyaluronan fragments activate an NF-kappa B/I-kappa B alpha autoregulatory loop in murine macrophages. J. Exp. Med. 1996, 183, 2373-2378.

75. Beck-Schimmer, B.; Oertli, B.; Pasch, T.; Wuthrich, R.P. Hyaluronan induces monocyte chemoattractant protein-1 expression in renal tubular epithelial cells. J. Am. Soc. Nephrol. 1998, 9, 2283-2290.

76. McKee, C.M.; Lowenstein, C.J.; Horton, M.R.; Wu, J.; Bao, C.; Chin, B.Y.; Choi, A.M.; Noble, P.W. Hyaluronan fragments induce nitric-oxide synthase in murine macrophages through a nuclear factor kappaB-dependent mechanism. J. Biol. Chem. 1997, 272, 8013-8018.

77. Sun, L.K.; Beck-Schimmer, B.; Oertli, B.; Wuthrich, R.P. Hyaluronan-induced cyclooxygenase-2 expression promotes thromboxane A2 production by renal cells. Kidney Int. 2001, 59, 190-196.

78. Hamerman, D.; Todaro, G.J.; Green, H. The production of hyaluronate by spontaneously established cell lines and viral transformed lines of fibroblastic origin. Biochim. Biophys. Acta 1965, 101, 343-351. 
79. Simpson, M.A.; Reiland, J.; Burger, S.R.; Furcht, L.T.; Spicer, A.P.; Oegema, T.R., Jr.; McCarthy, J.B. Hyaluronan synthase elevation in metastatic prostate carcinoma cells correlates with hyaluronan surface retention, a prerequisite for rapid adhesion to bone marrow endothelial cells. J. Biol. Chem. 2001, 276, 17949-17957.

80. Bullard, K.M.; Kim, H.R.; Wheeler, M.A.; Wilson, C.M.; Neudauer, C.L.; Simpson, M.A.; McCarthy, J.B. Hyaluronan synthase-3 is upregulated in metastatic colon carcinoma cells and manipulation of expression alters matrix retention and cellular growth. Int. J. Cancer 2003, 107, 739-746.

81. Tsuchiya, N.; Kondo, Y.; Takahashi, A.; Pawar, H.; Qian, J.; Sato, K.; Lieber, M.M.; Jenkins, R.B. Mapping and gene expression profile of the minimally overrepresented 8q24 region in prostate cancer. Am. J. Pathol. 2002, 160, 1799-1806.

82. Heldin, P.; Laurent, T.C.; Heldin, C.H. Effect of growth factors on hyaluronan synthesis in cultured human fibroblasts. Biochem. J. 1989, 258, 919-922.

83. Postlethwaite, A.E.; Smith, G.N., Jr.; Lachman, L.B.; Endres, R.O.; Poppleton, H.M.; Hasty, K.A.; Seyer, J.M.; Kang, A.H. Stimulation of glycosaminoglycan synthesis in cultured human dermal fibroblasts by interleukin 1. Induction of hyaluronic acid synthesis by natural and recombinant interleukin $1 \mathrm{~s}$ and synthetic interleukin 1 beta peptide 163-171. J. Clin. Invest. 1989, 83, 629-636.

84. Pienimaki, J.P.; Rilla, K.; Fulop, C.; Sironen, R.K.; Karvinen, S.; Pasonen, S.; Lammi, M.J.; Tammi, R.; Hascall, V.C.; Tammi, M.I. Epidermal growth factor activates hyaluronan synthase 2 in epidermal keratinocytes and increases pericellular and intracellular hyaluronan. J. Biol. Chem. 2001, 276, 20428-20435.

85. Yamada, Y.; Itano, N.; Narimatsu, H.; Kudo, T.; Morozumi, K.; Hirohashi, S.; Ochiai, A.; Ueda, M.; Kimata, K. Elevated transcript level of hyaluronan synthase1 gene correlates with poor prognosis of human colon cancer. Clin. Exp. Metastasis. 2004, 21, 57-63.

86. Kosaki, R.; Watanabe, K.; Yamaguchi, Y. Overproduction of hyaluronan by expression of the hyaluronan synthase Has2 enhances anchorage-independent growth and tumorigenicity. Cancer Res. 1999, 59, 1141-1145.

87. Itano, N.; Sawai, T.; Miyaishi, O.; Kimata, K. Relationship between hyaluronan production and metastatic potential of mouse mammary carcinoma cells. Cancer Res. 1999, 59, 2499-2504.

88. Enegd, B.; King, J.A.; Stylli, S.; Paradiso, L.; Kaye, A.H.; Novak, U. Overexpression of hyaluronan synthase-2 reduces the tumorigenic potential of glioma cells lacking hyaluronidase activity. Neurosurgery 2002, 50, 1311-1318.

89. Lokeshwar, V.B.; Rubinowicz, D.; Schroeder, G.L.; Forgacs, E.; Minna, J.D.; Block, N.L.; Nadji, M.; Lokeshwar, B.L. Stromal and epithelial expression of tumor markers hyaluronic acid and HYAL1 hyaluronidase in prostate cancer. J. Biol. Chem. 2001, 276, 11922-11932.

90. Franzmann, E.J.; Schroeder, G.L.; Goodwin, W.J.; Weed, D.T.; Fisher, P.; Lokeshwar, V.B. Expression of tumor markers hyaluronic acid and hyaluronidase (HYAL1) in head and neck tumors. Int. J. Cancer 2003, 106, 438-445.

91. Christopoulos, T.A.; Papageorgakopoulou, N.; Theocharis, D.A.; Mastronikolis, N.S.; Papadas, T.A.; Vynios, D.H. Hyaluronidase and CD44 hyaluronan receptor expression in squamous cell laryngeal carcinoma. Biochim. Biophys. Acta 2006, 1760, 1039-1045. 
92. Udabage, L.; Brownlee, G.R.; Nilsson, S.K.; Brown, T.J. The over-expression of HAS2, Hyal-2 and CD44 is implicated in the invasiveness of breast cancer. Exp. Cell Res. 2005, 310, 205-217.

93. Novak, U.; Stylli, S.S.; Kaye, A.H.; Lepperdinger, G. Hyaluronidase-2 overexpression accelerates intracerebral but not subcutaneous tumor formation of murine astrocytoma cells. Cancer Res. 1999, 59, 6246-6250.

94. Shuster, S.; Frost, G.I.; Csoka, A.B.; Formby, B.; Stern, R. Hyaluronidase reduces human breast cancer xenografts in SCID mice. Int. J. Cancer 2002, 102, 192-197.

95. Lokeshwar, V.B.; Cerwinka, W.H.; Isoyama, T.; Lokeshwar, B.L. HYAL1 hyaluronidase in prostate cancer: a tumor promoter and suppressor. Cancer Res. 2005, 65, 7782-7789.

96. Kuang, D.M.; Wu, Y.; Chen, N.; Cheng, J.; Zhuang, S.M.; Zheng, L. Tumor-derived hyaluronan induces formation of immunosuppressive macrophages through transient early activation of monocytes. Blood 2007, 110, 587-595.

97. de La Motte, C.A.; Hascall, V.C.; Calabro, A.; Yen-Lieberman, B.; Strong, S.A. Mononuclear leukocytes preferentially bind via CD44 to hyaluronan on human intestinal mucosal smooth muscle cells after virus infection or treatment with poly(I.C). J. Biol. Chem. 1999, 274, 30747-30755.

98. Day, A.J.; Prestwich, G.D. Hyaluronan-binding proteins: tying up the giant. J. Biol. Chem. 2002, 277, 4585-4588.

99. Zhuo, L.; Salustri, A.; Kimata, K. A physiological function of serum proteoglycan bikunin: the chondroitin sulfate moiety plays a central role. Glycoconj. J. 2002, 19, 241-247.

100. Milner, C.M.; Tongsoongnoen, W.; Rugg, M.S.; Day, A.J. The molecular basis of inter-alpha-inhibitor heavy chain transfer on to hyaluronan. Biochem. Soc. Trans. 2007, 35, 672-676.

101. Kida, D.; Yoneda, M.; Miyaura, S.; Ishimaru, T.; Yoshida, Y.; Ito, T.; Ishiguro, N.; Iwata, H.; Kimata, K. The SHAP-HA complex in sera from patients with rheumatoid arthritis and osteoarthritis. J. Rheumatol. 1999, 26, 1230-1238.

102. Shen, L.; Zhuo, L.; Okumura, A.; Ishikawa, T.; Miyachi, M.; Owa, Y.; Ishizawa, T.; Sugiura, N.; Nagata, Y.; Nonami, T.; Kakumu, S.; Kimata, K. The SHAP-hyaluronan complex in serum from patients with chronic liver diseases caused by hepatitis virus infection. Hepatol. Res. 2006, 34, 178-186.

103. Obayashi, Y.; Yabushita, H.; Kanyama, K.; Noguchi, M.; Zhuo, L.; Kimata, K.; Wakatsuki, A. Role of serum-derived hyaluronan-associated protein-hyaluronan complex in ovarian cancer. Oncol. Rep. 2008, 19, 1245-1251.

104. Kobayashi, N.; Miyoshi, S.; Mikami, T.; Koyama, H.; Kitazawa, M.; Takeoka, M.; Sano, K.; Amano, J.; Isogai, Z.; Niida, S.; Oguri, K.; Okayama, M.; McDonald, J.A.; Kimata, K.; Taniguchi, S.; Itano, N. Hyaluronan deficiency in tumor stroma impairs macrophage trafficking and tumor neovascularization. Cancer Res. 2010, 70, 7073-7083.

(C) 2011 by the authors; licensee MDPI, Basel, Switzerland. This article is an open access article distributed under the terms and conditions of the Creative Commons Attribution license (http://creativecommons.org/licenses/by/3.0/). 